\title{
The Influence of Surface Opening Crack on the Ultrasonic Waves Near the Surface in Concrete Members
}

\author{
Peng Wang ${ }^{1}$, Qing-bang Han ${ }^{2^{*}}$, Xue-ping Jiang ${ }^{3}$, Hao Zheng ${ }^{4}$ and Zhou-chen
} College of Internet of Things Engineering, Changzhou 213022, Jiangsu China

2hqb0092@163.com

Keywords: NDT, Concrete, Surface-opening Crack, Rayleigh Wave.

\begin{abstract}
Based on the velocities of waves near the surface in concrete, the propagation path and travel time of different waves were calculated, and the influence of crack on these waves was analyzed. Some theoretical conclusions were verified by FEM. The ultrasonic waves were received by array transducers and the velocities of different waves were calculated and distinguished according to travel time in the simulation. Simulation results show that when sound wave propagates through the crack, longitudinal waves arrive at the receiving arrays and the crack diffracts transverse wave to generate new longitudinal wave and transverse wave by mode conversion, and the Rayleigh wave propagates along the surface (including the crack side walls). Finally it is found that the receiving signal amplitude (energy) is approximately linearly monotone decreasing with crack depth.
\end{abstract}

\section{Introduction}

With the development of modern society, many engineering like motorway, bridges and dams demand lots of concrete whose bulk is huge. Affected by the leap of temperature, evaporation of water, undesirable construction method, however, it's easy to form opening cracks on the surface of concrete, which damage the structure. Therefore it's necessary to detect these cracks, and get hold of its depth and width to estimate the damage to the structure and the service life of the structure. There are many methods used in detecting opening cracks in concrete, such as: (1) single ultrasonic flat-measured method [6]; (2) perforating method; (3) Rayleigh wave method. The perforating method breaks structure, which costs too much; the single ultrasonic flat-measured method detects crack less than 500mm; however, the Rayleigh wave method has several advantages: (1) large energy; (2) not affected by fluid; (3) flexible detection for its propagation character.

Many researchers have done numerous researches on the detection of opening cracks of concrete. Based on the MFCE (Matched Filtering of Center of Energy), Foo Wei Lee [1] determined the arriving time of Rayleigh wave. G Hevin [2] put forward a function between the variation of spectral ratios between the transmitted and incident wave and the crack depth. Yang [3] combined the time reversal and the Rayleigh wave method, and found that enlarging the cracks depth resulted in an increase in the level of distortion in the reconstructed wave signals, and a higher damage index was obtained. Recently Zhang [4] obtained the relationship between the slot depth and the absorption frequency on the basis of spectral analysis.

Ultrasonic method has its advantages in detecting opening cracks, so it's necessary to analyze the influence of the opening cracks on the waves near surface including Rayleigh wave. Based on the sound velocities of different wave modes near the concrete surface, this paper analyzes the influence of opening crack on the ultrasonic waves near surface, and distinguishes them by utilizing receiving array. Simulation results show that longitudinal waves will arrive at the receiving array twice, and the crack will diffract transverse wave to generate new longitudinal wave and transverse wave by mode conversion, and Rayleigh wave will propagate along the surface (including the crack side walls), when sound waves near the surface propagate through the crack.

\section{Influence on the Ultrasonic Waves Near Surface}


The waves propagating along the surface are: longitudinal wave, transverse wave and Rayleigh wave. The velocity of longitudinal wave $\mathrm{cP}$ is fastest, and the velocity of transverse $\mathrm{cR}$ and Rayleigh wave $\mathrm{cS}$ are almost half of that of longitudinal wave. The ratio between $\mathrm{cR}$ and $\mathrm{cS}$ is dependent on the Poisson ratio. Here, the Poisson ratio of concrete is 0.33 , and the ratio of concrete is 0.93 .

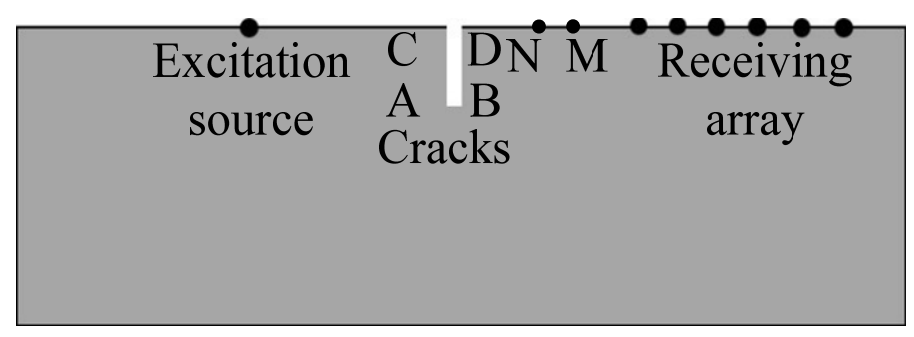

Figure 1. Model geometric structure.

On the basis of Fermat theorem, the Snell law near the crack walls and the Huyghens principle near the crack tips, this section analyzes the reflection and diffraction of waves caused by opening cracks, and computes the propagation path and time, and finally distinguishes the waves by velocity. Figure 1 shows the geometry of the structure, where $\mathrm{E}$ is the excitation source; $\mathrm{R}$ is the receiving array; $\mathrm{A}, \mathrm{B}$ are the left and right tips of the crack respectively; C, D are the two edges of the crack respectively; N, $\mathrm{M}$ are the mode conversion points. According to theories and the geometry, some analysis could be obtained:

(1) The longitudinal wave propagates fastest, and the original longitudinal wave is diffracted near the crack tips, afterwards the diffracted wave propagates to the receiving array at the velocity of longitudinal wave. This phenomenon is used in single ultrasonic flat-measured method [6]. This wave is denoted by $1 \mathrm{P}$-wave.

(2) According to (1), the original longitudinal wave is diffracted by crack tips, and the velocity of transverse wave caused by diffraction is slower, so the transverse wave will be converted into longitudinal wave by mode conversion when the wave propagates near the surface. This longitudinal wave is denoted by $2 \mathrm{P}$-wave.

(3) The original transverse wave is diffracted near the crack tips. The transverse wave caused by diffraction propagates into surface, and the mode conversion occurs at $\mathrm{N}$ point with the introduction of longitudinal wave and transverse wave at the same time. Among them, the longitudinal wave propagates at the soonest and is received by receiving array. The corresponding longitudinal is denoted by 3 P-wave.

(4) On the basis of (3), the mode conversion at $\mathrm{N}$ point introduces transverse wave and Rayleigh wave. The propagation time difference between them is small for the short propagation path and the similar velocity. The transverse wave is denoted by $1 \mathrm{~S}$-wave.

(5) As we know, Rayleigh wave propagates along the solid surface, so the propagation path of Rayleigh wave is along the surface from excitation source to the receiving array (including crack side walls). The Rayleigh wave is denoted by R-wave.

\section{Numerical Model and Results}

\section{Numerical Model}

FEM method is introduced to simulate the wave propagation near surface of two-dimension concrete, which is isotropous. Figure 2 shows the simulation geometry. 


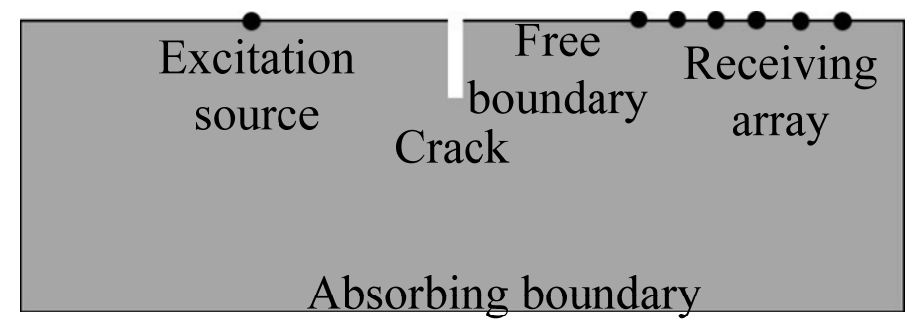

Figure 2. Model geometric.

The geometry size is $600 \mathrm{~mm} \times 200 \mathrm{~mm}$, filled with concrete; the parameters of the material are listed in Table 1. Correspondingly, $\mathrm{cP}=4013 \mathrm{~m} / \mathrm{s}, \mathrm{cS}=2021 \mathrm{~m} / \mathrm{s}, \mathrm{cR}=1886 \mathrm{~m} / \mathrm{s}$.

Table. 1 Medium parameter in the simulation

\begin{tabular}{llll}
\hline Solid acoustic & Young & Poisson & Density \\
constant & modulus & ratio & \\
value & $2.5 \times 10^{9}[\mathrm{~Pa}]$ & 0.33 & $2300\left[\mathrm{~kg} / \mathrm{m}^{3}\right]$ \\
\hline
\end{tabular}

In the simulation, the biggest size of mesh is $1.67 \mathrm{~mm}$; the time step is $4.16 \times 10-8 \mathrm{~s}$; sampling frequency is $50 \mathrm{MHz}$. Receiving array is adopted in the simulation which are at 400, 420, 440, 460, 480, 500mm denoted by R1, R2, R3, R4, R5, R6 respectively. Contacting surface between concrete and air is almost free boundary, and in order to avoid the disturbance of waves reflected by boundaries, absorbing boundary is adopted. For the sake of near field effect, Rayleigh wave and transverse wave are mixed, therefore the distance between transmit-receive probes couldn't be less than $150 \mathrm{~mm}$ according to document [5]. The distance selected in this paper is $200 \mathrm{~mm}$.

There are two simulation models for two purposes, one of which is to study the influence of crack on the waves, and the other is to study the relationship between crack depth and waves near surface with the depth of $20,30,40,50,60,70,80 \mathrm{~mm}$ respectively.

\section{The Influence of Crack on the Near Surface Wave}

Based on the parameters listed in Table 1, sound fields simulated by FEM are shown in Figure 3 and 4. By means of observing wave propagation and mode conversion in the sound field, the waves received by R1 are distinguished. Figure 5 shows the waves received by R1, and we can find the headmost three waves are longitudinal wave, and the next is slower transverse wave, and the final is Rayleigh wave.

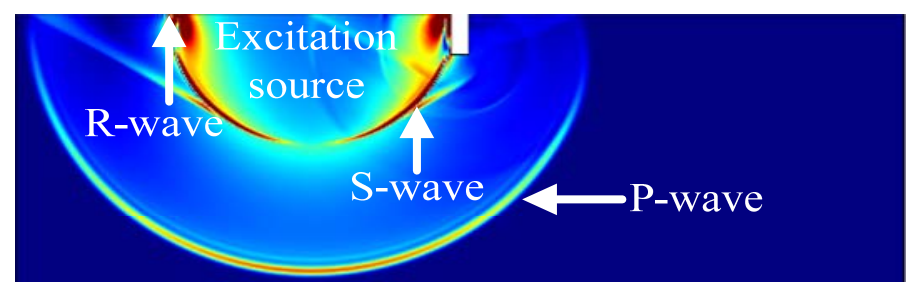

Figure 3. Sound field in $50 \mu$ s.

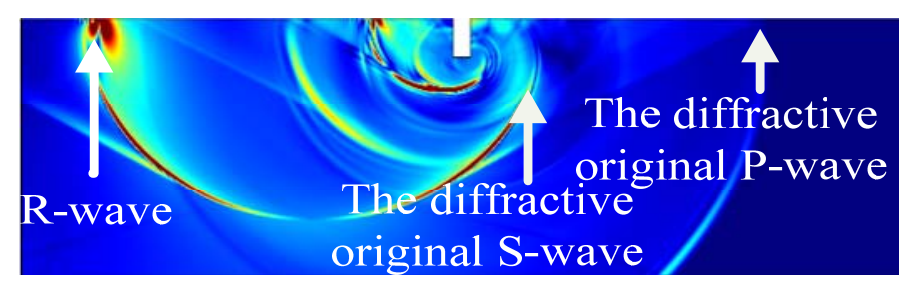

Figure 4. Sound field in $80 \mu$ s. 


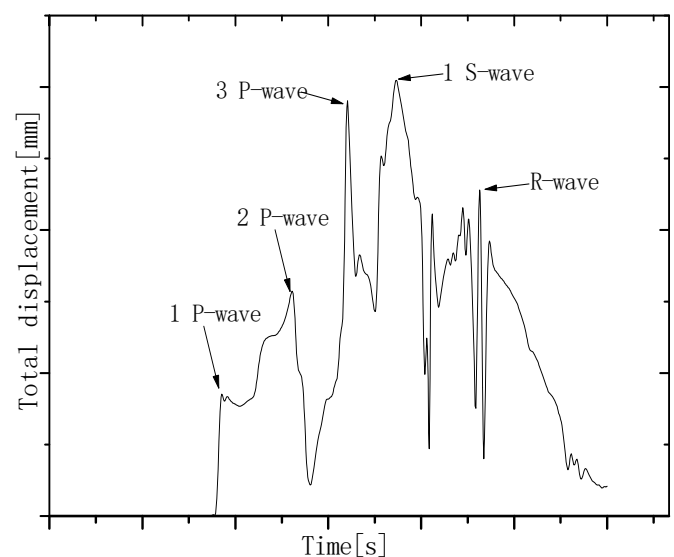

Figure 5. Waves near the surface received by $\mathrm{R} 1$ at $400 \mathrm{~mm}$.

To distinguish the modes of the received wave, the method of one transmitting and multi-receiving mode was adopted in the simulation. Figure 6 shows the wave signals received by R1 to R6.

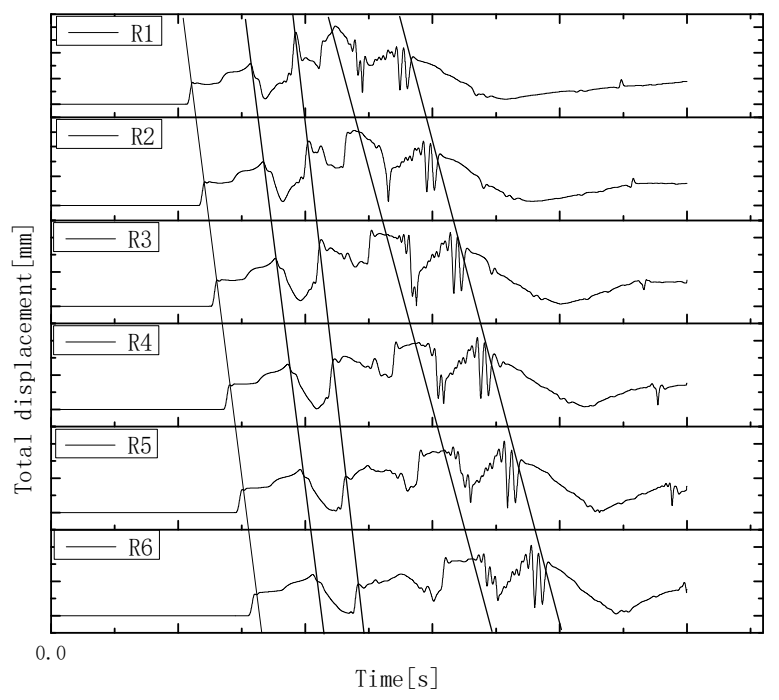

Figure 6. Waves near the surface received by R1, R2, R3, R4, R5, R6.

In Fig. 6, the oblique lines were adapted to link the arriving time of different waves for different receivers, the first three oblique lines indicate the velocities of longitudinal wave; the last two show transverse wave and Rayleigh wave respectively. Besides, the relationship between receivers and the arriving peak time of different waves is drawn in Figure 7.

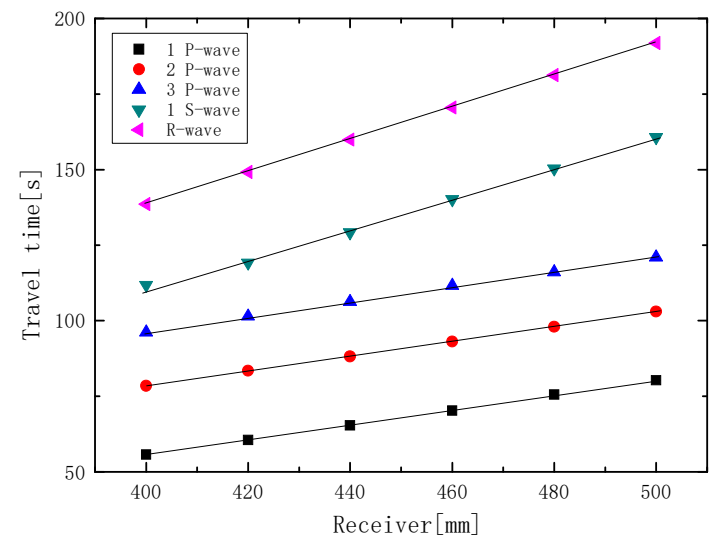

Figure 7. Sound velocity of different wave modes in the wave near surface 
According to Figure 7, the velocities of every wave are simulated that are: $\mathrm{cP}=4013 \mathrm{~m} / \mathrm{s}, \mathrm{cS}=2021 \mathrm{~s}$, $\mathrm{cR}=1886 \mathrm{~m} / \mathrm{s}$. The velocity of transverse wave is bit faster than the Rayleigh wave. In the sound field, the propagation times of different wave modes are simulated based on the propagation paths and velocities of different wave modes.

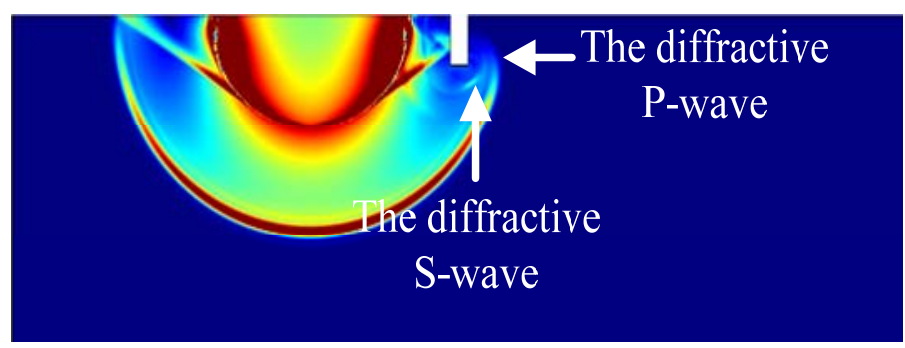

Figure 8. Sound field at $50 \mu \mathrm{s}$.

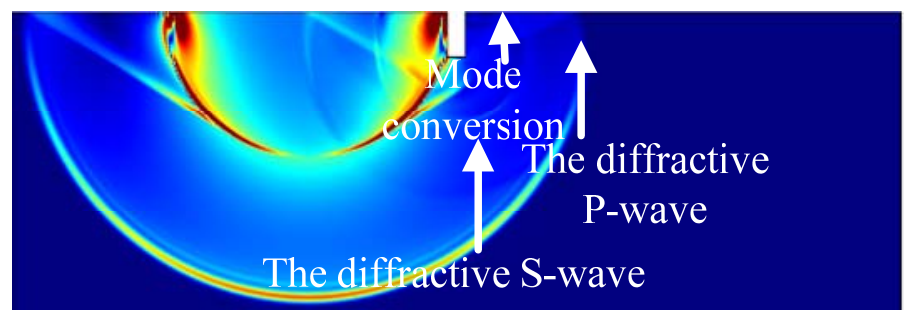

Figure 9. Sound field at $80 \mu$ s.

According to Figures 8 and 9, the original longitudinal wave is diffracted to introduce the new longitudinal wave and the new transverse wave. According to the Fermat theorem, the traveling time of the new longitudinal wave received by $\mathrm{R} 1$ is:

$$
t_{p 1}=\frac{(E A+A B+B R)}{c_{P}}=52.3 \mu \mathrm{s}
$$

The subscript $p$ indicates the longitudinal wave.

According to Figure 9, the new transverse wave introduced by the original longitudinal wave propagates to the concrete surface at a certain angle with the introduction of mode conversion. The point of mode conversion is approximately at $\mathrm{M}(375 \mathrm{~mm}, 200 \mathrm{~mm})$ according to the sound field. Therefore the traveling time of the second longitudinal wave ( $2 \mathrm{P}$-wave) received by $\mathrm{R} 1$ is:

$$
t_{p 2}=\frac{E A}{C_{P}}+\frac{A B}{C_{R}}+\frac{B M}{C_{S}}+\frac{M R}{C_{P}}=76 \mu \mathrm{s}
$$

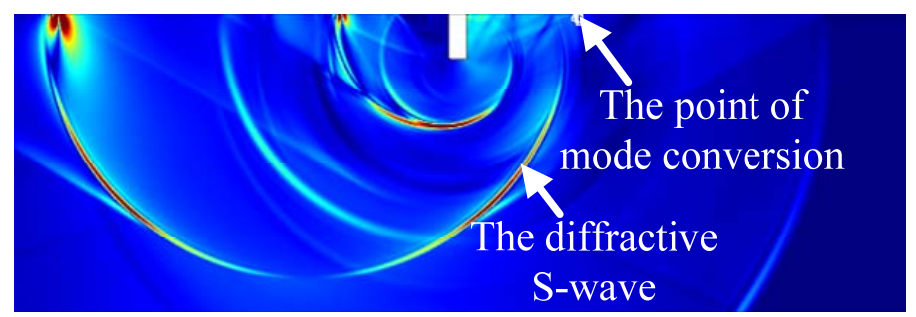

Figure 10. Sound field at $90 \mu \mathrm{s}$. 


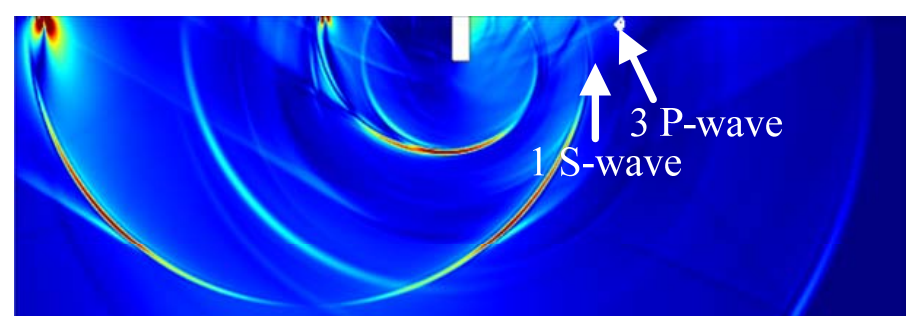

Figure 11. Sound field at $100 \mu \mathrm{s}$.

According to Figures 10 and 11, the original transverse wave was diffracted near the cracks tips, and the diffracted transverse wave which is dominating in the waves introduced by diffraction propagated to the concrete surface, with mode conversion similarly. The point of mode conversion is approximately at $\mathrm{N}(356 \mathrm{~mm}, 200 \mathrm{~mm})$ according to the sound field. Therefore the traveling time of the third longitudinal wave ( $3 \mathrm{P}$-wave) and the transverse wave received by $\mathrm{R}_{1}$ is:

$$
\begin{gathered}
t_{p 3}=\frac{E A}{c_{S}}+\frac{A B}{c_{R}}+\frac{B N}{c_{S}}+\frac{N R}{c_{P}}=96 \mu \mathrm{s} \\
t_{s 1}=\frac{E A}{c_{S}}+\frac{A B}{c_{R}}+\frac{B N}{c_{S}}+\frac{N R}{c_{S}}=106 \mu \mathrm{s} .
\end{gathered}
$$

The subscript $\mathrm{s}$ indicates the transverse wave.

Finally the slowest Rayleigh wave propagates along the surface including that of crack only, therefore the traveling time of Rayleigh wave received by R1 is:

$$
t_{r}=\frac{E C+C A+C B+B D+D R}{C_{R}}=137.9 \mu \mathrm{s} \text {. }
$$

The subscript $r$ indicates the Rayleigh wave.

The simulation results show that longitudinal wave arrives at the receiving array twice, and the crack diffracts transverse wave to generate new longitudinal wave and transverse wave due to mode conversion, and Rayleigh wave propagates along the surface (including the crack side walls), when sound waves near the surface propagate through the crack.

\section{The Relationship between Crack Depth and the Waves Near Surface}

To research the relationship between crack depth and the wave near surface, the crack width is $5 \mathrm{~mm}$; crack depths are 20,30, 40, 50,60,70, 80mm respectively; distance between transmit-receive probes is $200 \mathrm{~mm}$. Figure 12 and 13 show the wave signals received in the models with different crack depth.

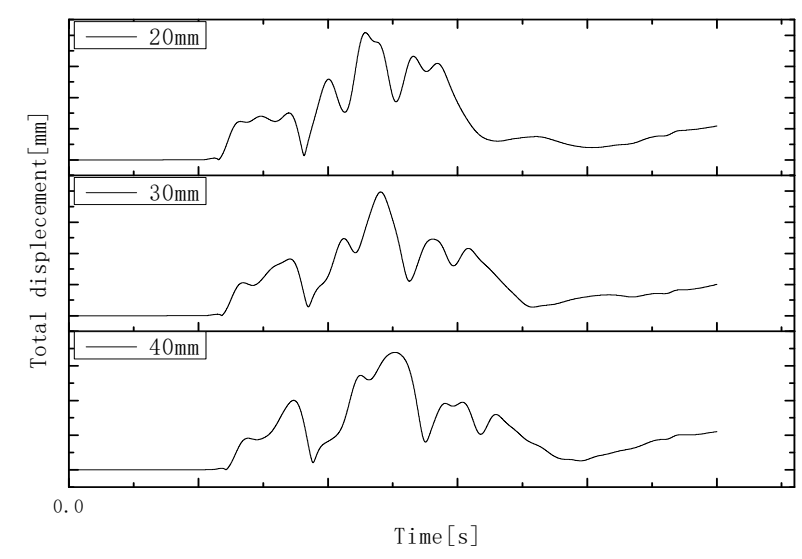


Figure 12. The wave shapes near surface in the model with the depth of 20, 30, $40 \mathrm{~mm}$.

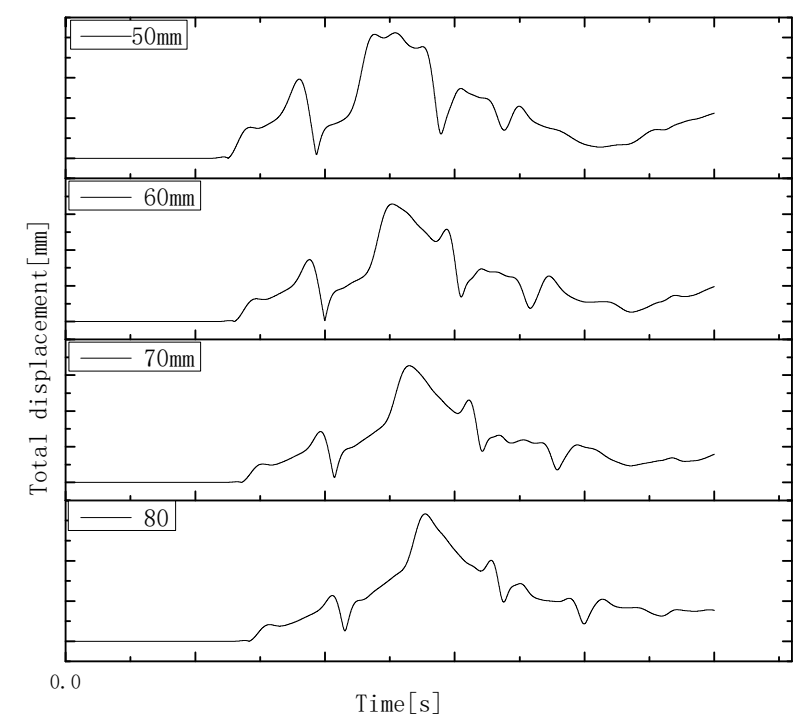

Figure 13. The wave shapes near surface in the model with the depth of 50,60, 70, $80 \mathrm{~mm}$.

Based on the data in Figures 12 and 13, the relationship between crack depth and the wave near surface could be obtained: Figure 14 shows the relationship between crack depth and the amplitude of $1 \mathrm{P}$-wave and figure 15 shows the relationship between crack depth and the energy of $1 \mathrm{P}$-wave and 2 P-wave.

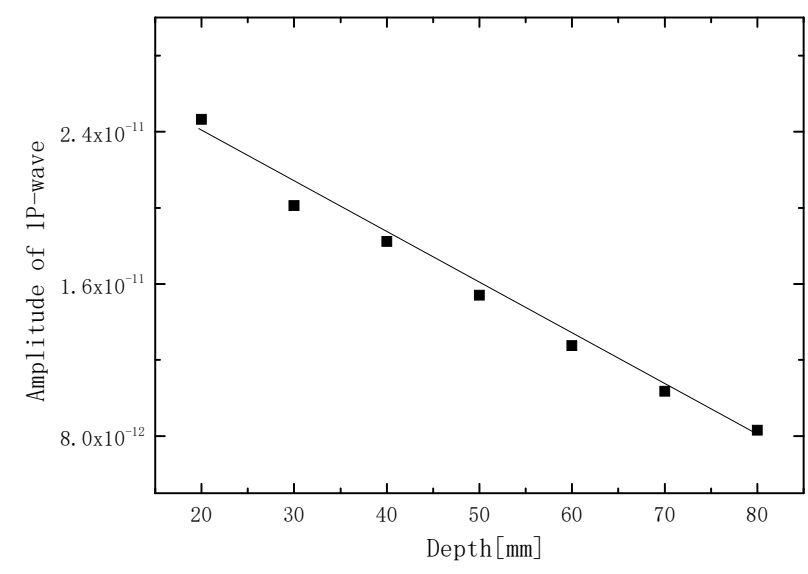

Figure 14. The crack depth versus the amplitude of $1 \mathrm{P}$-wave.

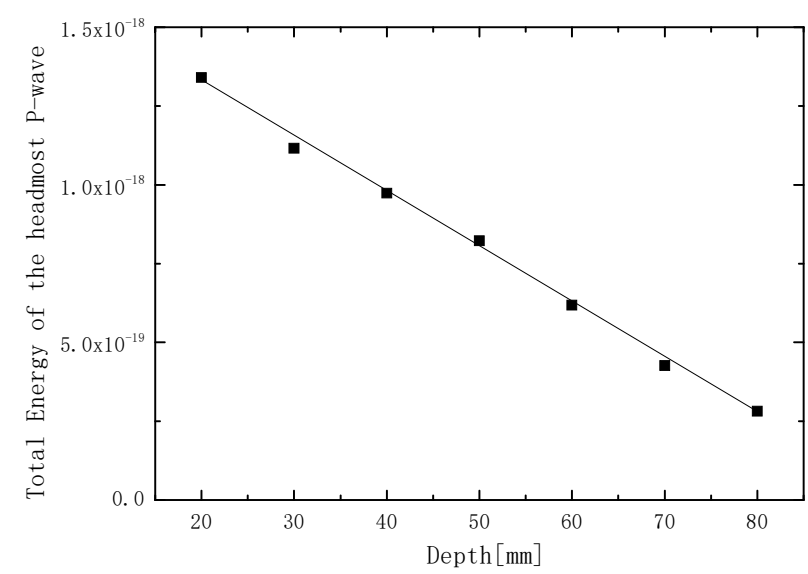

Figure 15. The relationship between the crack depth and the energy of 1, 2 P-wave. 
The propagation path of wave increases with the increase of crack depth, and the amplitude and energy of wave decrease. The relationship between crack depth and the amplitude of first longitudinal or the energy of the former two longitudinal waves is approximately linear monotone decreasing.

\section{Conclusions}

The influence of the opening crack on the waves near surface is analyzed in this paper. Simulation results show that when sound wave propagates through the crack, longitudinal wave arrives at the receiving arrays, and the crack diffracts transverse wave to generate new longitudinal wave and transverse wave by mode conversion, and Rayleigh wave propagates along the surface (including the crack side walls). Finally, it is found that the receiving signal amplitude (energy) is approximately linearly monotone decreasing with crack depth.

\section{Acknowledgement}

This work was supported by the Natural Science foundation of China Grant No11574072 and 11274091 , Key research project of Jiangsu Grant No BE2016056.

\section{References}

[1] Lee F W, Lim K S, Chai H K, Determination and extraction of Rayleigh-waves for concrete cracks characterization based on matched filtering of center of energy. Journal of Sound and Vibration, 2016, 363: 303-315.

[2] Hevin G, Abraham O, Pedersen H A, et al, Characterization of surface cracks with Rayleigh waves: a numerical model. NDT \& E International, 1998, 31(4): 289-297.

[3] YANG Y, ZHANG M Y, XIAO L, QU W Z, Simulation study of surface breaking crack in concrete beam based on time-reversed theory of surface wave. Concrete, 2015 (12): 17-20.

[4] ZHANG Z D, LU C, WEI Y F, Numerical simulation to Sizing surface-breaking Cracks by ultrasonic Surface Wave spectral Method. Nondestructive Testing, 2009 (12): 993-997.

[5] FENG R Y, CHEN Y, LI Z S, Cracks identification of mass concrete structures with R wave spectral energy transmission ratio method. Journal of vibration and shock, 2016, 35(12): 221-225.

[6] 2000C. Technical specification for inspection of concrete defects by ultrasonic methods. 